\title{
Recent developments in ion detection techniques for Penning trap mass spectrometry at TRIGA-TRAP
}

\author{
J. Ketelaer ${ }^{1, a}$, K. Blaum ${ }^{2,3}$, M. Block ${ }^{4}$, K. Eberhardt ${ }^{5}$, M. Eibach ${ }^{1}$, R. Ferrer ${ }^{1, b}$, S. George ${ }^{1,2}$, F. Herfurth $^{4}$, \\ J. Ketter ${ }^{1}$, Sz. Nagy ${ }^{1,2}$, J. Repp ${ }^{1}$, L. Schweikhard ${ }^{6}$, C. Smorra ${ }^{3,5}$, S. Sturm ${ }^{1}$, and S. Ulmer ${ }^{1,3}$ \\ 1 Institut für Physik, Johannes Gutenberg-Universität Mainz, Staudinger Weg 7, D-55128, Germany \\ 2 Max-Planck-Institut für Kernphysik, Saupfercheckweg 1, D-69117 Heidelberg, Germany \\ 3 Physikalisches Institut, Ruprecht-Karls-Universität Heidelberg, Philosophenweg 12, D-69120 Heidelberg, Germany \\ 4 GSI Helmholtzzentrum für Schwerionenforschung GmbH, Planckstraße 1, D-64291 Darmstadt, Germany \\ 5 Institut für Kernchemie, Johannes Gutenberg-Universität Mainz, Fritz-Strassmann-Weg 2, D-55128 Mainz, Germany \\ ${ }^{6}$ Institut für Physik, Ernst-Moritz-Arndt-Universität Greifswald, Felix-Hausdorff-Straße 6, D-17489 Greifswald, Germany
}

Received: 10 September 2008 / Revised: 6 December 2008

Published online: 3 February 2009 - (C) Società Italiana di Fisica / Springer-Verlag 2009

Communicated by J. Äystö

\begin{abstract}
The highest precision in the determination of nuclear and atomic masses can be achieved by Penning trap mass spectrometry. The mass value is obtained through a measurement of the cyclotron frequency of the stored charged particle. Two different approaches are used at the Penning trap mass spectrometer TRIGA-TRAP for the mass determination: the destructive Time-Of-Flight Ion Cyclotron Resonance (TOF-ICR) technique and the non-destructive Fourier Transform Ion Cyclotron Resonance (FT-ICR) method. New developments for both techniques are described, which will improve the detection efficiency and the suppression of contaminations in the case of TOF-ICR. The FT-ICR detection systems will allow for the investigation of an incoming ion bunch from a radioactive-beam facility on the one hand, and for the detection of a single singly charged ion in the Penning trap on the other hand.
\end{abstract}

PACS. 07.75. $+\mathrm{h}$ Mass spectrometers - 07.50.Ek Circuits and circuit components - 07.50.Qx Signal processing electronics - 07.77.Ka Charged-particle beam sources and detectors

\section{Introduction}

High-precision mass measurements are relevant to many fields in physics. The required mass uncertainties $\delta \mathrm{m} / \mathrm{m}$ range from $10^{-6}$ to below $10^{-11}$, depending on the application [1]. The highest precision in mass measurements is presently obtained with Penning traps, where a charged particle is stored in a superposition of a strong homogeneous magnetic field and a weak electric quadrupole field. The motion of an ion inside a Penning trap is well understood [2]. It consists of two radial eigenmotions, the modified cyclotron and the magnetron motion with corresponding eigenfrequencies $\left(\nu_{+}, \nu_{-}\right)$, and an axial oscillation $\left(\nu_{z}\right)$. From an independent measurement of these eigenfrequencies the cyclotron frequency

$$
\nu_{c}=\frac{1}{2 \pi} \frac{q}{m} B
$$

\footnotetext{
${ }^{a}$ e-mail: ketela@uni-mainz.de

b Present address: National Superconducting Cyclotron Laboratory, MSU, East Lansing, MI 48824-1321, USA.
}

can be extracted by means of the invariance theorem [3]

$$
\nu_{c}^{2}=\nu_{+}^{2}+\nu_{z}^{2}+\nu_{-}^{2}
$$

which can be simplified for an ideal Penning trap to the relation $\nu_{c}=\nu_{+}+\nu_{-}$. Here, $q$ denotes the charge state, $m$ the mass of the stored particle, and $B$ the magnetic field.

There are presently two techniques to determine the cylotron frequency in a Penning trap: for very short-lived radionuclides with half-lives down to $10 \mathrm{~ms}$ [4] and production rates of only 100 ions/s [5], the time-of-flight ion cyclotron resonance (ToF-ICR) method $[6,7]$ is widely used $[1,8]$. The ions in the trap, which move on a defined magnetron orbit, are excited by a quadrupolar rf field at frequency $\nu_{r f}$ in the plane perpendicular to the magnetic field direction. Thereby, the two radial eigenmotions are coupled, in case $\nu_{r f}=\nu_{+}+\nu_{-}$. Since $\nu_{+} \gg \nu_{-}$, the radial energy is mainly determined by the modified cyclotron motion. After excitation, the ions are ejected out of the trap and reach a particle detector outside the strong $B$ field after they passed the gradient of the magnetic field. Thereby, the radial energy is transformed into axial energy 
and the time of flight from the trap to the detector has a minimum for a resonant excitation at $\nu_{r f}=\nu_{c}$. Typically, at least 100 ions are needed for a single resonance. For the application of this method, highly efficient particle detectors are needed. In the past, mainly micro-channel plates (MCP) [9] have been used, which can have a comparably large detection surface. However, only a fraction of about $60 \%$ is sensitive to incoming ions due to the channel structure of this detector type. Thus, the detection efficiency is already limited. A detector setup has recently been introduced at ISOLTRAP [10] allowing the use of either an MCP or a channeltron electron multiplier (CEM) [11]. The channeltron reaches almost hundred percent detection efficiency with a conversion dynode. Secondary electron multipliers are limited by the incoming beam current, i.e. the number of ions in a certain pulse length $[12,13]$. In typical applications the detector has to be operated at beam currents below $10 \mathrm{pA}$ to avoid saturation, corresponding to about 100 ions in a $1 \mu$ s pulse.

A crucial point in TOF-ICR measurements is the identification of events that contain contaminations. Unresolved isobaric or isomeric contaminants cause systematic shifts in the cyclotron frequency of the nuclide of interest [7, 14]. A typical ratio between wanted and unwanted species in the precision trap that can be still handled by further cleaning in a ToF-ICR measurement is about 1:1. Recently, a position-resolving MCP detector has been applied for the first time as a time-of-flight detector. Simulations and first tests showed that the position information on the detector can be used to identify the contaminations in the resonance spectrum [15]. This information can be used to reject all events with contaminants present in the trap. Another field of application for the position information is the identification of isomeric states.

A non-destructive detection method based on the image currents of ions in the trap electrodes is known as FT-ICR [16]. This technique is a standard method in analytical chemistry to investigate large ion clouds with typically 1000 or more ions with a comparably low, i.e. ppm, precision. FT-ICR detection is also used at high-precision mass spectrometers for stable isotopes, e.g. MiT-TRAP (now: FSU, Tallahassee, Florida, USA) [17,18] and the Uw-MPIK-Ptms (Washington, USA, now: MPI-K, Heidelberg, Germany) [19,20], and in Penning trap facilites to determine magnetic moments $[21,22]$. In the field of highprecision mass measurements on short-lived radionuclides, some of the interesting nuclides have only very low production rates in radioactive-beam facilities, sometimes less than one per minute. In these cases, single-ion sensitivity is needed which can only be reached by the narrow-band FT-ICR. In order to get a sufficiently long transient a minimum half-life of about $1 \mathrm{~s}$ of the ion is required. Especially superheavy nuclides often provide both low production rates and comparably long half-lives. While the time for a TOF-ICR resonance would extend to more than a day due to the low production rates and the need for a repeated loading of the trap, a resonant FT-ICR pick-up needs only a single ion to determine the cyclotron frequency, assuming sufficient storage time is possible for the given half-life. Moreover, a broad-band version of this technique can be applied to investigate the ion cloud coming from an online source and to identify contaminants, which can then be cleaned away by mass-selective excitation.

The mentioned techniques will be implemented at the TRIGA-TRAP double-Penning trap mass spectrometer [23] recently installed at the research reactor TRIGA Mainz (Germany) [24]. This setup serves as a test bench for the new methods that will be applied to other on-line mass spectrometers like SHIPTRAP (GSI, Darmstadt, Germany) [25]. Moreover, samples of actinide elements like ${ }^{254} \mathrm{Cf}$ produced off-line can be investigated, which are ideal test candidates on the way to transactinide nuclides $(Z \geq 104)$ as produced by SHIP (GSI) [26]. The Penning trap setup will later be also coupled to the TRIGA reactor to investigate neutron-rich fission products. In the following, the development of the different detection techniques at TRIGA-TraP will be discussed.

\section{Detectors for time-of-flight ion cyclotron resonance measurements}

The work at TRIGA-TrAP focuses on two points concerning the optimization of charged-particle detectors for TOF-ICR mass measurements: i) the detection efficiency should be optimized to reach a value close to $100 \%$, and ii) the position information of the particle on the detector should be utilized as an additional criterion to identify contaminants. Both approaches will pave the way for time-of-flight mass measurements on radionuclides with production rates of about 1 particle per second, even if a large background of contaminations is present.

\subsection{Micro-channel plate and channeltron}

MCP and CEM are commonly used for the ion detection, which are both based on the read-out of secondary electron avalanches triggered by charged particles. MCPs consist of many channels arranged in a honeycomb structure. At Triga-TraP the MCP for the time-of-flight detection has been chosen with a diameter such that it covers the complete cross-section of the drift tubes from the Penning trap to the detector. The drawback of MCP detectors is the comparably low efficiency for low-energy ion beams. A typical value is about $30-50 \%$ for ion impact energies around $2 \mathrm{keV}$ [27]. However, for higher ion impact energies $(\approx 4-6 \mathrm{keV})$ MCPs can reach detection efficiencies of $60-86 \%[28,29]$.

A CEM with conversion dynode has been recently introduced at the Penning trap mass spectrometer Isoltrap (Isolde, CERN) [11]. This type of CEM is mounted off-axis to the incoming ion beam so that neutral particles cannot reach the detector. The dynode is used to convert the incoming ion beam into electrons with a conversion efficiency of almost $100 \%$. The electron energy can be easily tuned by the potential difference applied between the dynode and the front surface of the CEM detector. Thereby, the total detection efficiency of this type of detector is $\geq 90 \%$ independent from the energy of the incoming 
ions. At TrigA-TrAP, a combined setup including both ion detectors (MCP and CEM) similar to IsOLTRAP will be used for the time-of-flight measurement allowing for a fast exchange during a run. The detection efficiencies have been compared using a pulsed ion beam with a fixed number of ions per pulse [11]. This test showed an efficiency gain of a factor of $>3$ for a CEM with conversion dynode in comparison to an $\mathrm{MCP}$ for ions in the low-energy range $(\leq 100 \mathrm{eV})$. In this measurement, the MCP was operated at a front voltage of $-2.1 \mathrm{kV}$ and the CEM at $-2 \mathrm{kV}$ with the conversion dynode at $-4 \mathrm{kV}$. Therefore, the ion impact energy in case of the MCP and the electron impact energy for the CEM were about $2 \mathrm{keV}$. These are typical operation parameters in ToF-ICR setups.

\subsection{Position-resolving ion detection}

So far, only the time-of-flight information has been used for TOF-ICR measurements. Recently, a position-resolving MCP has been introduced to obtain the coordinates of the ion impact on the detector surface [15]. Compared to a standard MCP, the anode is replaced by a delay line wire grid. This grid is formed by two coils with axes perpendicular to each other but parallel to the detector surface. An incident particle triggers a secondary electron avalanche in the channels at the impact point. These avalanches proceed to the delay line grid and cause an electric current moving on each of the coils to both wire ends. The position information is determined by the time difference of the arrival of the current signal on the different ends and the signal progressing velocity parallel to the detector surface. With the commercial detector DLD40 from RoentDek in use at TRIGA-TRAP a spatial resolution of about $70 \mu \mathrm{m}$ can be achieved [30].

Simulations show that the position of an ion in the trap corresponds to a specified impact position on the detector. Thereby, the radial ion motion in the Penning trap can be monitored in a ToF-ICR measurement (see fig. 1). As already mentioned, the ToF-ICR method is based on the determination of the cyclotron frequency by observing the ions' time of flight as a function of the excitation frequency $[6,7]$. The stored ions are first excited at the almost mass-independent magnetron frequency $\nu_{-}$, to increase the magnetron radius $\rho_{-}$for all ions present in the trap, prior to the coupling of the two radial eigenmotions by the quadrupolar field with the frequency $\nu_{r f}=\nu_{c}$. The latter leads to a periodic conversion between magnetron and cyclotron motion for the ion species in resonance with $\nu_{r f}$. After a full conversion, $\rho_{-}$is zero but the cyclotron radius $\rho_{+}$is now as large as the inital $\rho_{-}$. Due to the high frequency $\nu_{+}$the ions are equally distributed over all cyclotron phases by the ejection pulse, which is in the order of a few micro-seconds. By imaging the ion position in the trap on the position-resolving time-of-flight detector for several ejection pulses, the non-excited ions should form a single spot corresponding to the phase-locked magnetron motion, whereas the excited ones distribute over a circle corresponding to the cyclotron radius $\rho_{+}$. Therefore, contaminations which did not get excited in their motional

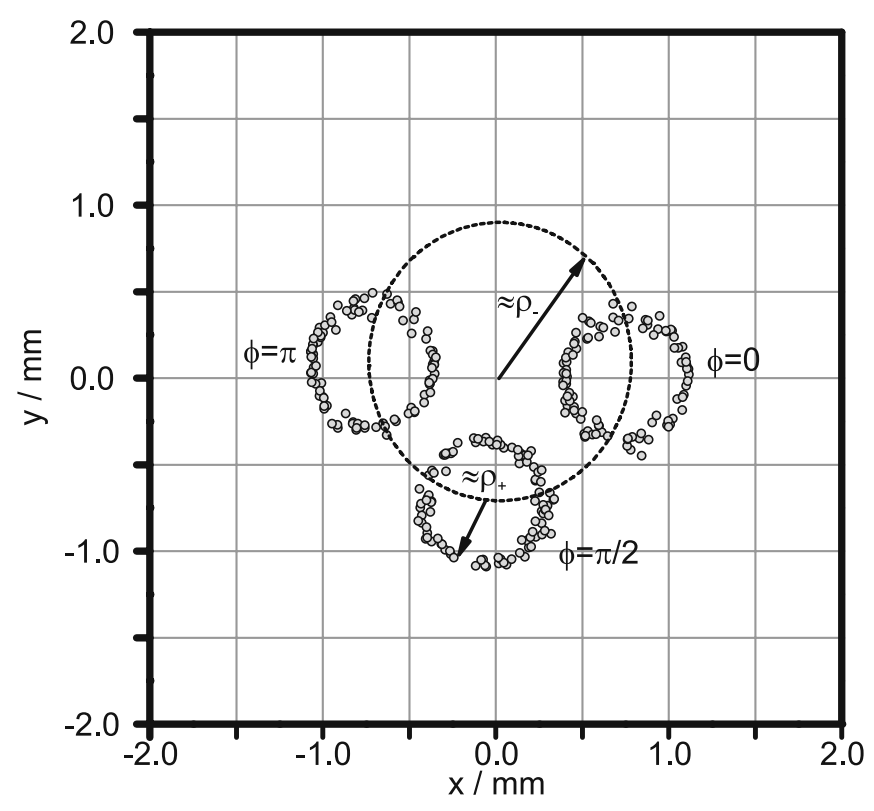

Fig. 1. Simulation of the ion ejection from a Penning trap at three different magnetron phases $\phi$. The cyclotron phase cannot be determined due to the high frequency $\nu_{+}$of this eigenmotion. The ions form a circle with cyclotron radius $\rho_{+}$. The center depends on the magnetron phase and moves on a circle with magnetron radius $\rho_{-}$.

amplitudes can be identified and removed from the ToFICR spectrum. This is of particular interest in cases where isobaric or isomeric contaminations are as abundant in the Penning trap as the ions of interest. Systematic shifts in the determined cyclotron frequency would result in case all ions in a pulse with a contaminant in the trap would not be rejected.

The capability of the position-resolving MCP detector to monitor the ion position in the Penning trap and to identify contaminations has been demonstrated in offline experiments at the SHIPTRAP facility [15]. The two isotopes ${ }^{85} \mathrm{Rb}$ and ${ }^{87} \mathrm{Rb}$ (relative abundance about 2.5:1) from a surface ion source have been used in a ToF-ICR measurement at the cyclotron frequency of ${ }^{87} \mathrm{Rb}^{+}$as described above after they have been prepared with zero magnetron radius. Figure 2 shows the results: the excited ions (in this case ${ }^{87} \mathrm{Rb}^{+}$) occupy a cyclotron orbit, whereas the non-excited ions $\left({ }^{85} \mathrm{Rb}^{+}\right)$stay in their magnetron motion, which is visible as a single spot on the detector plane. A measure for the quality of a time-of-flight resonance is the contrast

$$
C=\frac{t_{\text {nonexc }}-t_{\text {exc }}}{\sqrt{\sigma_{\text {nonexc }}^{2}+\sigma_{\text {exc }}^{2}}},
$$

where $\left(t_{e x c}, \sigma_{e x c}\right)$ are the mean time of flight and the standard deviation for the excited ions, and $\left(t_{\text {nonexc }}, \sigma_{\text {nonexc }}\right)$ for the non-excited ions, respectively. The mean time of flight for all recorded events in this figure is $t=(67.3 \pm$ 6.3) $\mu \mathrm{s}$. Without the position information, this would be taken for $\left(t_{e x c}, \sigma_{e x c}\right)$. All events within the detector area selected by the box have $t=(71.1 \pm 2.3) \mu$ s corresponding to $\left(t_{\text {nonexc }}, \sigma_{\text {nonexc }}\right)$. Taking the position information into 

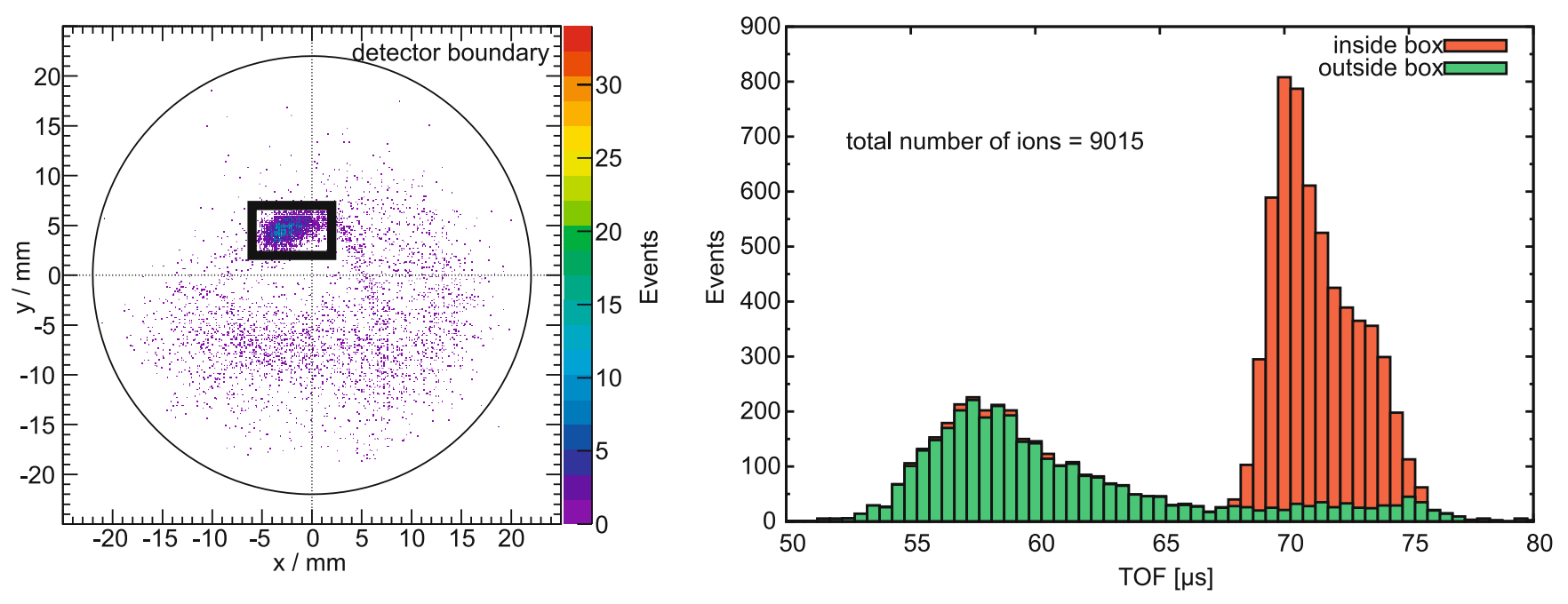

Fig. 2. (Colour on-line) Position-resolved ion detection in an off-line experiment at SHIPTRAP (GSI) with the two isotopes ${ }^{85}$ Rb and ${ }^{87} \mathrm{Rb}$. In the $x-y$ plot on the left, the position information for each incidenting particle is diplayed. The right plot shows the corresponding time-of-flight distribution. The total height of each column represents the total counts for the corresponding time-bin. The fraction inside and outside the box is marked in color. For more details see text.

account, the distribution of the excited ions outside the box leads to $t=(61.1 \pm 5.8) \mu$ s. In this special case, the time-of-flight contrast in resonance for the excited ${ }^{87} \mathrm{Rb}^{+}$ ions could be improved by a factor of 3 .

\section{Non-destructive ion detection: FT-ICR}

A limiting factor for ToF-ICR mass measurements is the production rate of the nuclide of interest at radioactivebeam facilities. Especially in the region of heavy and superheavy elements, some nuclides can only be produced at a rate of one per minute or even less. Taking the typical total efficiency of an on-line Penning trap mass spectrometer into account which is in the order of $1-5 \%$, this leads to less than one detected event per hour. A typical minimum number of ions required for a single ToF-ICR mass measurement is about 100. Measurement runs over several days needed in this case to acquire the statistics are not feasible due to long-term drifts and fluctuations of the magnetic field and the electric potentials.

At this point, the non-destructive FT-ICR detection technique can be applied. Among the actinide and transactinide nuclides, some species yield rather long half-lives which are in the order of a few seconds or even longer. This enables single-ion measurements as well as repeated measurement cycles with the same trap content to improve the statistics. FT-ICR is based on the detection of the image current induced by the ions in the trap electrodes [16]. The statistical uncertainty of the mass value in an FT-ICR measurement is given by

$$
\frac{\delta m}{m} \approx \frac{1}{\nu_{c} T \sqrt{N}},
$$

where $\nu_{c}$ denotes the cyclotron frequency of the ion, $T$ is the observation time, and $N$ the number of recorded transients. The expression has been originally derived for
TOF-ICR resonances in [31]. For a typical cyclotron frequency of $1 \mathrm{MHz}$, an observation time of $1 \mathrm{~s}$, and 10 transients, the uncertainty would be a few times $10^{-7}$. The transient length and the number of transients are strongly limited by the half-life and the production rate of the ion of interest, respectively.

There are many detection schemes possible focussing on the different eigenfrequencies. At Triga-Trap, the ring electrodes of both Penning traps are four-fold segmented with two $40^{\circ}$ segments for the excitation and two $140^{\circ}$ segments for the detection of the image current signal. In the following, two different approaches of the further signal processing are discussed.

\subsection{Broad-band FT-ICR}

The purification trap of TRIGA-TrAP is a cylindrical Penning trap used for buffer gas cooling and isobaric separation [32]. Therefore, the ion bunch coming from the ion source is stored and mass independently excited to a larger magnetron radius. In the next step, a quadrupolar rf field at the true cyclotron frequency of the ions of interest is applied to convert the magnetron motion into cyclotron motion similar to the excitation for a TOF-ICR measurement. The buffer gas causes the cyclotron radius to decrease very fast, whereas the ions in the magnetron motion stay on their orbit. This is due to the much lower collison rate in the low-frequency magnetron motion. Only the centered ions can pass an orifice behind the trap leading to a mass-selective ion ejection. To investigate the composition of the ion cloud coming from the source, this procedure has to be repeated while scanning the cyclotron excitation frequency.

A more elegant way to obtain information on the ion species present in the cloud is the broad-band FT-ICR technique. At Triga-Trap the two larger segments of the ring electrode will be used for the image current detection 
(a)

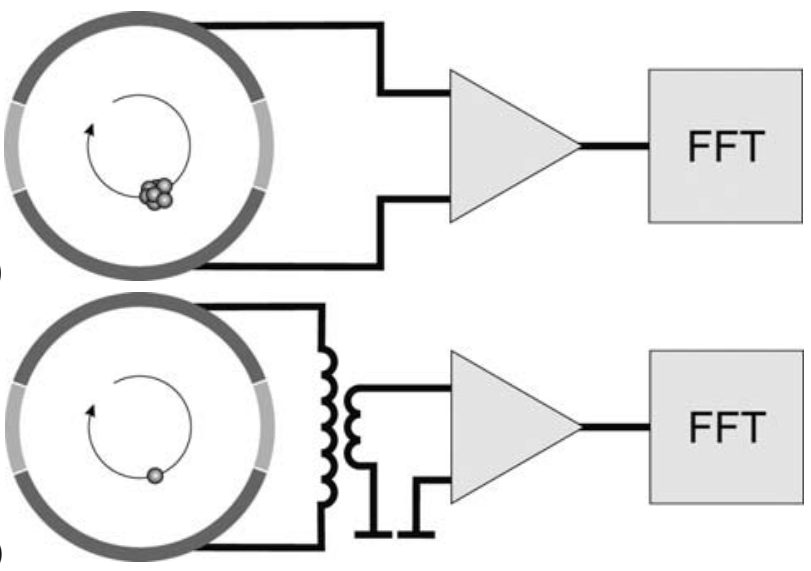

Fig. 3. Principle of the image current detection at TRIGATRAP with the FT-ICR method. (a) For the broad-band detection of an ion cloud, the signal from two opposite $140^{\circ}$ segments of the ring electrode of the purification trap is directly amplified. (b) For the single-ion detection, the two ring segments of the precision trap are connected to a superconducting tank circuit. In both cases, the signal is amplified and a fast Fourier transformation (FFT) is carried out. For more details see text.

(see fig. 3(a)). The image current generates a voltage drop across the parasitic capacitance of the trap electrodes, which is amplified. A spectrum analyzer performs the fast Fourier transformation (FFT) revealing the frequency information. The buffer gas pressure in the purification trap of about $10^{-6}$ mbar limits the sampling time for each transient to about $10 \mathrm{~ms}$. Therefore, the resolving power is $\leq 10^{4}$ for an ion frequency of $1 \mathrm{MHz}$.

Several commercial amplifiers have been tested for this purpose concerning noise properties and input capacitance as well as input resistance. Broad-band FT-ICR has several demands on the amplifiers to be used: a high input resistance ensures a flat frequency response of the circuit. The input capacitance adds to the parasitic capacitance of the trap electrodes and the wires.

Two amplifiers seem to meet the requirements best and will be tested in the TrIGA-Trap setup: the Stanford Research Systems SR560 [33] has a voltage noise of about $4 \mathrm{nV} / \sqrt{\mathrm{Hz}}$ in the frequency range from $0.1-1 \mathrm{MHz}$ and a current noise of about $2 \mathrm{pA} / \sqrt{\mathrm{Hz}}$ at a gain of 1000 and a frequency of $100 \mathrm{kHz}$. The input capacitance has been determined to be in the order of $50 \mathrm{pF}$, the input resistance is $100 \mathrm{M} \Omega$. This device has two channels which can be also used in differential mode. The other candidate is the SA-220F5 from NF [34] with a voltage noise of only $0.4 \mathrm{nV} / \sqrt{\mathrm{Hz}}$ in the frequency range from $10 \mathrm{kHz}-10 \mathrm{MHz}$ and a current noise of $68 \mathrm{fA} / \sqrt{\mathrm{Hz}}$ at $500 \mathrm{kHz}$. The input capacitance was determined to be $65 \mathrm{pF}$ and the input resistance $1 \mathrm{M} \Omega$.

As an example the signal-to-noise ratio for the SA-220F5 calculated with the test results given above is shown as a function of the frequency in fig. 4 . For the signal amplitude, a coherent motion of $N=1000$ ions in the modified cyclotron degree of freedom has been assumed. The ratio between the radius of the ion orbit and the char-

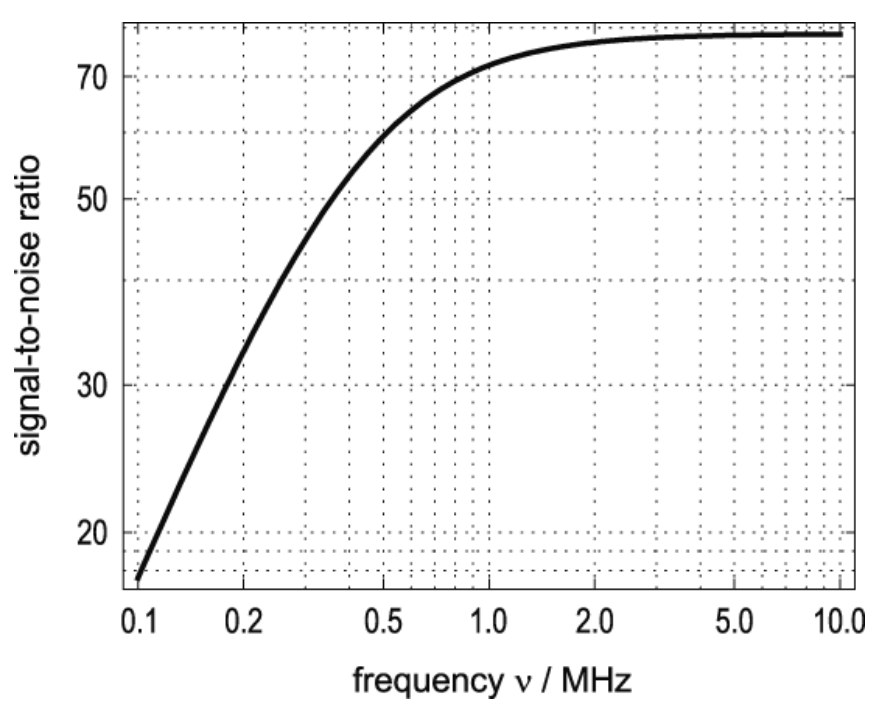

Fig. 4. Signal-to-noise ratio calculated from the measured current and voltage noise densities, as well as the input impedance and capacitance of the SA-220F5 amplifier. A coherent motion of 1000 ions at an orbit with a radius of 0.05 times the characteristic trap dimension has been assumed for the signal amplitude.

acteristic trap dimension is $\rho_{+} / d=0.05$. For a totally incoherent motion, the signal-to-noise ratio is lower by a factor of $\sqrt{N}$. The frequency dependence for the SR560 is similar, but the absolute signal-to-noise ratio is about a factor of 10 less.

To improve the signal-to-noise ratio even further, a cryogenic amplifier is currently being tested off-line, which will be operated close to the purification trap in a $77 \mathrm{~K}$ environment.

\subsection{Single-ion sensitivity}

Single-ion sensitivity enables the measurement of a full frequency spectrum with a single ion. Moreover, repeated measurements on the same stored singly charged ion will become possible at TRIGA-TRAP to accumulate the statistics. For this purpose a narrow-band FT-ICR detection system has been developed.

The aim of this image current detection is to improve the signal-to-noise ratio by reducing the detection bandwidth to a narrow window centered around the ion motion frequency. At Triga-TraP, a superconducting NbTi coil is connected to the two larger segments of the ring electrode of the precision trap (see fig. 3(b)), which forms an LC resonance circuit in combination with the parasitic capacitance of the detection electrodes and the wire. The quality factor $Q$, defined as the ratio between the center frequency and the width at $3 \mathrm{~dB}$ from the maximum, is about 15000 in the unloaded case. The helical coil is cooled to $4.2 \mathrm{~K}$ in a liquid-helium cryostat outside the magnet housing the Penning traps, which are at $77 \mathrm{~K}$. This arrangement is a compromise due to the limited diameter of the room temperature bore of the superconducting 


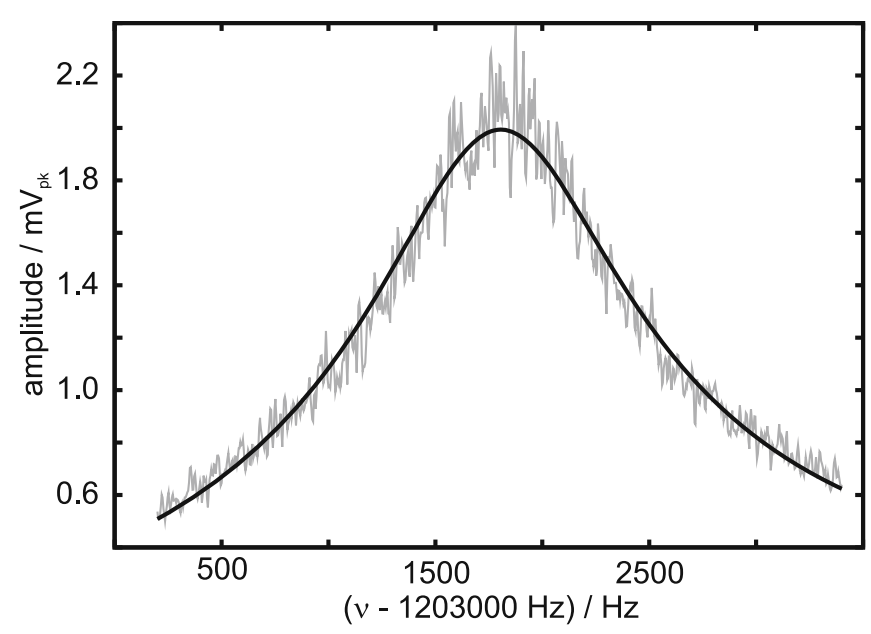

Fig. 5. Resonance of the superconducting helical coil for the narrow-band FT-ICR detection connected to the precision trap via a set of varactor diodes to tune the center frequency. The loaded $Q$-value has been determined for this special tuning of the resonance frequency to be $Q=(1004 \pm 19)$. The solid line is a fit of the theoretical line shape.

magnet and the required possibility of ion injection and ejection for simultaneous TOF-ICR measurements. Due to vacuum feedthroughs and the circuit needed to tune the resonance frequency, the $Q$-value decreases to about 1000 when the coil is connected to the trap (see fig. 5). The inductivity of the coil is $(337 \pm 6) \mu \mathrm{H}$ leading to a resonance frequency in the order of $1.2 \mathrm{MHz}$, which can be tuned to match the two stable rubidium isotopes ${ }^{85} \mathrm{Rb}$ and ${ }^{87} \mathrm{Rb}$ for first tests. The signal-to-noise ratio at the coil is given by

$$
\frac{S}{N} \approx \sqrt{\frac{I_{\text {ion }}^{2} G_{\text {cryo }}^{2} \hat{R}_{p}^{2} \gamma^{2}}{\left[\left(4 k_{B} T \hat{R}_{p} \gamma^{2}+u_{\text {cryo }}^{2}\right) G_{\text {cryo }}^{2}+u_{r t}^{2}\right] \Delta \nu}},
$$

where

$$
I_{i o n}=\sqrt{2} \pi \frac{\rho_{+}}{D} q \nu_{+}
$$

is the induced ion current [35] with the radius $\rho_{+}$and the frequency $\nu_{+}$of the modified cyclotron motion. $D$ is the effective distance between the ring segments used for the detection, $G_{\text {cryo }}$ the gain of the cryogenic amplifier attached to the coil, $T$ the temperature of the circuit, $u_{\text {cryo }}$ and $u_{r t}$ are the voltage noise densities for the cryogenic and the first room temperature amplifiers. $\gamma=k \times N_{2} / N_{1} \times \sqrt{l_{1} / l_{2}}$ is composed of the winding ratio $\left(N_{1} / N_{2}\right)$, the length ratio $l_{1} / l_{2}$, and the coupling $k$ between the primary and secondary side of the coil;

$$
\hat{R}_{p}=\frac{Q}{2 \pi \nu_{+} C} \frac{R_{\text {cryo }} / \gamma^{2}}{Q /\left(2 \pi \nu_{+} C\right)+R_{\text {cryo }} / \gamma^{2}}
$$

is the effective parallel resistance given by the total capacitance $C$ of the circuit attached to the primary side of the coil and the input resistance $R_{\text {cryo }}$ of the cryogenic amplifier. $\Delta \nu$ denotes the detection bandwidth. Presently, we are constructing another superconducting coil for the detection of actinide ions. With this coil a signal-to-noise ratio of about $2-5$ is estimated for a single singly charged ion of mass $A=250 \mathrm{u}$.

The FT-ICR signal will be coupled to a $4.2 \mathrm{~K}$ amplifier via a secondary winding of the superconducting coil. Two room temperature circuits with operational amplifiers with a total gain of about $4.5 \times 10^{5}$ will also be used. The signal will be band-pass filtered and heterodyned prior to the transient recording by a NI PCI-4551 card from National Instruments.

\section{Conclusions}

Penning trap mass spectrometry faces new challenges when exploring the transactinides and the nuclides far away from stability. A number of isotopes of heavy and superheavy elements have rather long half-lives in the order of seconds or longer. In these cases, the new developments concerning non-destructive ion detection at TRIGA-TRAP will help to overcome the limit of very low production rates by enabling single-ion sensitivity and repeated measurements on the same stored ion. For very short-lived nuclides, the TOF-ICR method is still the tool of choice since the measurement cycle is fast enough to deal with the short half-lives. The position-resolving time-of-flight detection improves the selectivity of this technique concerning further suppression of background events. The methods and techniques discussed here will be first applied and tested at the TRIGA-TRAP Penning trap mass spectrometer in Mainz, prior to an implementation at other facilities like SHIPTRAP.

We acknowledge financial support by the Helmholtz Association for National Research Centers (HGF) under contract VH-NG-037, as well as by the Stiftung Rheinland-Pfalz für Innovation under contract 854 . Sz. Nagy acknowledges financial support by the Humboldt Foundation. We also acknowledge the support from S. Stahl.

\section{References}

1. K. Blaum, Phys. Rep. 425, 1 (2006).

2. L. Brown, G. Gabrielse, Rev. Mod. Phys. 58, 233 (1986).

3. L. Brown, G. Gabrielse, Phys. Rev. A 25, 2423 (1982).

4. M. Smith, M. Brodeur, T. Brunner et al., arXiv preprint, arXiv: 0807.1260.

5. A. Kellerbauer, G. Audi, D. Beck et al., Phys. Rev. Lett. 93, 72502 (2004).

6. G. Gräff, H. Kalinowsky, J. Traut, Z. Phys. A 297, 35 (1980).

7. M. König, G. Bollen, H. Kluge et al., Int. J. Mass Spectrom. Ion Process. 142, 95 (1995).

8. L. Schweikhard, G. Bollen (Editors), Ultra-Accurate Mass Spectrometry and Related Topics, Int. J. Mass Spectrom. 251, issue nos. 2-3 (2006) (special issue).

9. J. Wiza, Nucl. Instrum. Methods 162, 587 (1979).

10. M. Mukherjee, D. Beck, K. Blaum et al., Eur. Phys. J. A 35, 31 (2008).

11. C. Yazidjian, K. Blaum, R. Ferrer et al., Hyperfine Interact. 173, 181 (2006). 
12. BURLE: Channeltron ${ }^{T M}$ Electron Multiplier Handbook for Mass Spectrometry, http://www.burle.com/cgi-bin/ byteserver.pl/pdf/ChannelBook.pdf (2001).

13. S. Coeck, M. Beck, B. Delauré et al., Nucl. Instrum. Methods A 557, 516 (2006).

14. A. Kellerbauer, K. Blaum, G. Bollen et al., Eur. Phys. J. D 22, 53 (2003).

15. G. Eitel et al., submitted to Nucl. Instrum. Methods (2008).

16. A. Marshall, C. Hendrickson, G. Jackson, Mass Spectrom. Rev. 17, 1 (1998).

17. S. Rainville, J. Thompson, D. Pritchard, Science 303, 334 (2004).

18. W. Shi, M. Redshaw, E. Myers, Phys. Rev. A 72, 22510 (2005).

19. R. Van Dyck jr., S. Zafonte, S. Van Liew et al., Phys. Rev. Lett. 92, 220802 (2004).

20. D. Pinegar, S. Zafonte, R. Van Dyck, Hyperfine Interact. 174, 47 (2007).

21. G. Werth, J. Alonso, T. Beier et al., Int. J. Mass Spectrom. 251, 152 (2006).

22. B. Odom, D. Hanneke, B. D'Urso et al., Phys. Rev. Lett. 97, 30801 (2006).
23. J. Ketelaer, J. Krämer, D. Beck et al., Nucl. Instrum. Methods A 594, 162 (2008).

24. K. Eberhardt, A. Kronenberg, Kerntechnik 65, 269 (2000).

25. M. Block, D. Ackermann, K. Blaum et al., Eur. Phys. J. A 45, 39 (2007).

26. G. Münzenberg, W. Faust, S. Hofmann et al., Nucl. Instrum. Methods 161, 65 (1979).

27. B. Brehm, J. Grosser, T. Ruscheinski et al., Meas. Sci. Technol. 6, 953 (1995).

28. H.C. Straub, M.A. Mangan, B.G. Lindsay et al., Rev. Sci. Instrum. 70, 4238 (1999).

29. A. Müller, N. Djurić, G.H. Dunn et al., Rev. Sci. Instrum. 57, 349 (1986).

30. RoentDek Handels GmbH, Kelkheim Ruppertshain, http://www.roentdek.de.

31. G. Bollen, Nucl. Phys. A 693, 3 (2001).

32. G. Savard et al., Phys. Lett. A 158, 247 (1991).

33. Stanford Research Systems, Inc., 1290-D Reamwood Avenue, Sunnyvale, CA 94089, USA, http://www . thinksrs . com.

34. NF Corporation, 6-3-20 Tsunashima Higashi, Kohoku-ku, Yokohama 223-8508, Japan, http://www.nf corp.co.jp.

35. L. Schweikhard, Int. J. Mass. Spectrom. 107, 281 (1991). 